\title{
Sentiment Analysis of Health Care: Review
}

\author{
Issam AATTOUCHI ${ }^{*}$, Saida ELMENDILI ${ }^{2 *}$, Fatna ELMENDILI ${ }^{3}$
}

\author{
${ }^{1}$ Computer science research Laboratory, Faculty of Sciences, Ibn Tofail University, Kenitra, Morocco \\ 2 Engineering Sciences Laboratory, National School of Applied Sciences, Ibn Tofail University, Kenitra, \\ Morocco \\ ${ }^{2}$ Image laboratory, Moulay Ismail University of Meknes, School of Technology, Meknes, Morocco
}

\begin{abstract}
Twitter is a microblogging service where users can send and read short messages of 140 characters called "tweets". Many healthcare-related unstructured and free-text tweets are shared on Twitter, which is becoming a popular domain for medical research. Sentiment analysis is one of the data mining types that provides an estimate of the direction of personality sentiment analysis in natural language processing. By analyzing text, computational linguistics is used to infer and analyze mental knowledge of the web, social media, and related references. The data reviewed actually quantifies the attitudes or feelings of the global society towards specific goods, people, or thoughts and exposes the contextual duality of the knowledge. Sentiment analysis is used in various sectors such as health care. There is an incredible amount of healthcare information available online, such as social media, and websites focused on rating medical problems, that is not accessed in a methodical way. Sentiment analysis has many benefits, such as using medical information to achieve the best possible patient outcome and improve the quality of health care. This review paper focuses on the presented sentiment analysis methods that are used in the medical field.
\end{abstract}

\section{Introduction}

Sentiment analysis (SA), also known as opinion extraction, is a branch of natural language processing (NLP) that classifies sentiment in free text automatically. Its roots may be traced back to the 1990s, with methods for viewpoints classification [1], prediction of adjectives semantic orientation [2], subjectivity classification [3], and so on. However, the arrival of Web 2.0 and the everincreasing availability of user-generated data, such as product and service evaluations, as well as the proliferation of social networking platforms, have all contributed to its rapid expansion.

SA has been found to be useful in a range of societal settings, including industry, economy, and government [4-8].

This study focuses on health apps, which are defined as "a condition of complete mental, bodily, and social wholeness, rather than simply the absence of diseases or infirmities" [9]. Well-being is defined as a perceived or subjective condition, which means that it can differ significantly amongst individuals in otherwise similar circumstances [10].

This fact makes well-being an optimal case study for SA. Regarding health issues, of course, Because of this, wellbeing is an excellent case study for SA. Of course, when it comes to health issues, modern society is focused on negative occurrences like illness, damage, and incapacity [11], making SA difficult in this field. For example, having a good quality of life for a patient with a long-term illness will depend less on the absence of accompanying problems and more on how well they are controlled and managed.

The unfavorable complication of health symptoms, on the other hand, tends to shift the SA's outcomes towards the poor end of the range.

Today, both doctors and patients use online platforms such, social media, and websites to express their view on health issues [12]. Informatics is defined as "the science of information dissemination and transmission in an online medium, notably the Internet, or in a population, with the overriding purpose of influencing public health and public policy" [13]. Real-time data collection and processing is possible from social media such as Twitter, with the potential to survey public opinion (sentiment) on a given topic [14]. Social media has been described by Bates and colleagues as a "perfect storm" for patientfocused healthcare, which provides a valuable source of data for the public and healthcare institutions [15]. Twitter is part of this, as it is easy to use, cheap, and accessible. There are 955 million followers on Twitter today, which is a mobile microblogging and social networking service. There are actually 955 million registered users on Twitter who can share post containing text, videos, photos or links to external sources. Fully one-third of people with social media profiles use Twitter, and $75 \%$ of them access it from a mobile device to express an opinion $[16,17]$.

Sentiment analysis examines the content of natural language in free text, i.e., the individual words and signs used in a text message, to determine the intensity of positive and negative opinions and feelings. Sentiment analysis using social media is a widely studied topic already [18]. It is useful for corporate marketing to

\footnotetext{
* Corresponding author: elmendili.saida@uit.ac.ma
} 
comprehend the opinions of the public or of consumers about their product [19]. Computer-based software tools have been generated to automate the sentiment analysis process, quickly transforming large numbers of free-text reviews into quantitative sentiment scores, e.g., positive or negative [18]. They are usually based on text classifiers or machine learning based processes. They tend to be business oriented, expensive, and focus on capturing opinions about a specific chosen product or department [20]. During the H1N1 influenza outbreak, Chew et al performed a tweet content analysis [21]. In this research, they provided a qualitative and categorical measure of sentiment using content classifiers such as "humor" or "sarcasm." Accurately analyzing sentiment in an automated manner is challenging due to the subjectivity, flexibility, and creativity of the language used [22]. The purpose of this study was to examine the methods used to measure sentiment for Twitter-based healthcare studies.

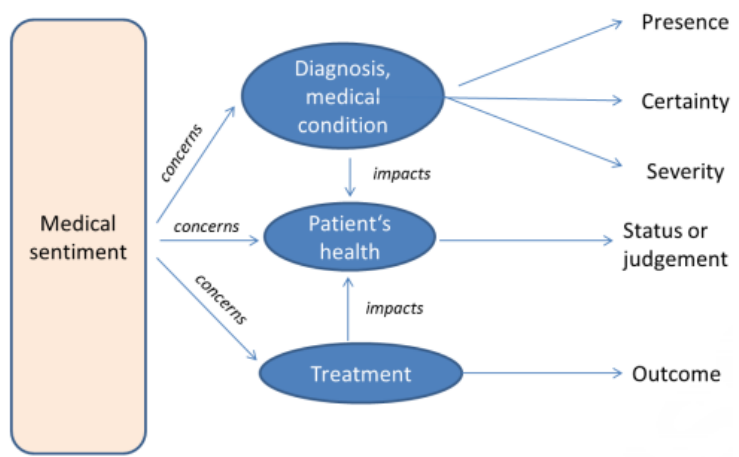

The first objective was to examine which methods of sentiment analysis were used and in what healthcare context. The second objective was to examine the extent to which the methods were practiced and validated for the study data, and whether a rationale for their use was provided.

Fig. 1 Facets of sentiment in medical contexts.

\section{METHODS}

Methods for determining and analyzing subjectivity, as well as opinions and sentiments presented in textual form, have been created in the field of web science and web browsing. This field is also known as sentiment analysis or opinion extraction. In medicine and health care, sentiment analysis is of growing interest.

\subsection{General overview}

Sentiment analysis research began in 2004 [23] with the investigation of customer comments and online news for opinions. The challenge at hand was to discern between positive and negative viewpoints, as well as subjective and objective sections of a document. Later on, additional tasks were added: emotion analysis determines the emotional category of texts [24,25], whereas intensity focuses on detecting the many levels of policies or feelings (e.g., very positive, very sad).
However, these methods can be used to identify the degree of sensitivity of the text and to identify the level of emotion (e.g., very positive, very sad).

The classification problem is addressed in existing sentiment analysis systems built for processing unstructured text in Web media: a classifier is trained to determine the level of polarity at the sentence or document level. Pang et al., for example, proposed a number of supervised methods, including support vector machines (SVM), naive Bayes classification, and maximum entropy $[26,27]$.

A solution for recognizing the text semantic focus has been developed by Turney, who suggested an unsupervised approach [28]. These applications are based on feature sets that include semantic or lexical features.

The main principle is that the feeling is expressed through opinion statements and is expressly mentioned in the text. Adjectives, adverbs, and particular nouns, on the other hand, usually indicate sentiment in free writing.

Therefore, traditional sentiment analysis is based on the analysis and detection of these opinion words (semantic features). Using a lexical survey, these are then analyzed by sentiment analysis algorithms to find opinion terms and their policies in a document.

In addition to lexicon lookup to find opinion terms, other features can be extracted from texts to be processed by sentiment analysis methods. These include lexical attributes such as unigrams, bigrams, and words in parts of speech. Sentiment analysis can be done at several levels, including word, aspect, phrase, and document.

Pang et al [29] considered the problem at the document level. However, there is a great need for more detailed methods of sentiment analysis. Aspect-based analysis seeks to identify elements of a feature and attribute a sentiment to each one (for example, the CD is good but it was too valuable describes two aspects, the CD's content and its price) [30]. Sentiment analysis has been found to be Domain-dependent [31], as the polarity of certain phrases varies depending on the context in which they are employed. Therefore, the feature models on what a machine learning classifier relies on must be trained on the specific domain datasets. Lexicons must be appropriate for field-specific understandings of words. A domain neutral approach to sentiment analysis was introduced by Montejo-Raez et al [32]. They displayed each term in a tweet as a vector of factored WordNet words that are semantically near to the term.

SentiWordNet [33] employed the individual weights to produce polarity estimate.

There isn't a lot of research on analyzing feelings from a text-based perspective. Balahur et al. used sentiment analysis methodologies to filter out excellent and bad news information from good and poor sentiment supplied about a target, as well as to explicitly mark the opinions [34]. 
For this special type of text that is the news article, this is to some extent similar to the analysis of sentiments in the domain of health: sentiments can be transmitted by medical diseases, courses of treatment or health conditions, and they have an effect on the patients' health status and quality of life.

\subsection{Sentiment lexicons}

Many of the methods used in the sentiment analysis are now based on the use of sentiment vocabularies, which provide an excellent basis for the analysis of sentiment in the recognition of words and phrases in a language. They are an important basis for the identification of expressions and speech patterns of sentiment in natural language texts. Some examples of existing lexicons available include SentiWordNet (SWN) [33], WordNetAffect, General Inquirer1, and the Sentiment Lexicon (SL) [35].

They both have words in them and assign sentiment scores or categories to them. Lexicographers can create lexicons by hand or by studying corpora. They frequently ignore the nuances and differing meanings of terminology when employed across domains, or they fail to provide explicit information about other subjects. One of the most widely used sentiment lexicons is SentiWordNet.

It attributes to each WordNet synset three feelings scores: positivity, negativity, and objectivity.SentiWordNet has a variety of word definitions. However, it is not specified in which domain a phrase has a different meaning (For example, if right is simply a navigational term or a term that means "correct"). Word NetAffect [36] assigns one or more affective labels to a group of WordNet synsets that represent emotional remarks.

Mohammad and Turney [36] have built EmoLex, a manually created emotion lexicon using Amazon's Mechanical Turk service. The Lexicon of Subjectivity by Wilson et al [35] includes 8221 unique term expressions of subjectivity combined with their related polarity. In order to use these global feelings resources, field-specific vocabulary will be further developed based on the word's meanings domain specificity.

The most common domain words, along with their highlevel semantic linkages and event patterns, would be included in a multidimensional high-quality domain lexicon. Enhancing an existing vocabulary is the most direct way to cover the domain-specific environment.

Combining a generic lexicon with a domain-specific lexicon is one way to improve the lexicon's coverage. While traditional methods have mostly focused on finding the explicit expression of sentiment, Deng et al. [37] provided an annotation technique that took into account both active beneficial and active masculine occurrences across opinion entities, resulting in an extension of the well-known Subjectivity Lexicon (MPQA) [35]. In particular, four types of "good for" and "bad for" (gfbf) of events were developed: destruction, generation, gain or loss, benefit or injury. Events are expressed as a text triplet: agent, gfbf, object, which indicated that a subject (noun) such as a person or organization had a good or adverse effect on the object (noun).

The author's suggested viewpoints are listed in the figure. Goeuriot et al. [38] combined words from SentiWordNet and Subjectivity Lexicon [35], two general-purpose lexicons. The merged lexicons were then supplemented with opinion words from medical reviews.

While extending, the differences in terms of polarity between the General and Medical fields were examined. The authors discovered that in pharmacological publications, some terms that are generally thought to be neutral are actually bearers of opinion. Finally, using a basic scoring system, it was showed that using the combined vocabulary yielded better results than using well-known broad lexicons of sentiment. Sentitivity Lexicon [35], General Inquirer [20], SentiWordNet, and Moby were used by Ohana et al [39] to assess the accuracy of feeling recognition. Text is from six categories of general domain datasets, such as hotel reviews, book reviews, movies and conversations, was added to the lexicons. The findings revealed that the level of accuracy of individual Lexicons varied by domain.

SentiWordNet, in instance, had the best accuracy (65$71 \%$ ) in four of the six domains, while the subjective lexicon had the greatest performance $(63-65 \%)$ in the remaining two. Specifically, SentiWordNet achieved the best accuracy (65-71\%) in four of the six fields, and the Subjectivity Glossary performed best $(63-65 \%)$ in the other two domains.

SentiWordNet's extensive vocabulary coverage was one aspect in this.

\subsection{Sentiment analysis in a medical setting}

In terms of generic strategies for analyzing attitudes, the following sections go over the various approaches in further depth.

In the field of medicine, sentiment analysis is categorized according to the textual source (e.g., medical web content, biomedical publications, clinical notes), the method (e.g., based on polarity, classification, rules, machine learning), and type of analysis (outcome classification), and level (e.g., word level, sentence level). The most important methods are detailed in the section that follows.

\subsection{Sentiment Analysis from the Medical Web}

In existing research, feeling is often considered as a polarity, i.e. a positive, negative or neutral polarity towards a given subject. Such a categorization is relevant when, in

This categorization is relevant when, in texts, opinions are expressed about a person (e.g., a doctor), a drug, or a medical device. However, the sentiment may be even more important.

As opposed to people or products, where feelings primarily include appreciation or dislike of a person or 
good, views or feelings about medications, treatments, or even diagnoses

have even more faces and are phrased in many different words:

- Opinions about medical doctors, medical devices, and medications,

- Personal sentiments about one's own or others' health conditions,

-Complications that have occurred,

- Facts and experiences about a particular treatment, diagnosis or medication.

A treatment can be painful, but useful. Complications may have occurred, but the treatment was still successful. A diagnosis can be frightening, but not really serious or fatal. A medication can have serious side effects. How can we characterize feeling in medical texts?

It is clear that sentiment or opinion in medical social media can be expressed differently than sentiment in news or product reviews. A sentiment can also be described by a symptom that reflects a person's health condition. It is not just a feeling, it is characterized by symptoms, by pathological terms. Since in

social media, a mixture of facts and experiences is expressed, it may be necessary to distinguish factual information from experiences.

Most sentiment analysis work in the field of medicine is focused on Web data such as medical blogs or discussion groups, with the goal of mining or studying the opinions of individual patients or to measure the quality of healthcare.

As an example, an approach was implemented to split evidence-based texts from evidence-based texts [45] in order to evaluate the accuracy and validity of the patient generated content. With the assumption that content that is factual is actually better than affective content, as it is more informative (as opposed to moods and experience). With the concept that factual material is better than emotive material since it provides more information, a system was created to evaluate factual material in medical social media utilizing subjective terms and a medical ontology. Existing approaches to sentiment analysis from medical online data are typically machine learning-based [45] or rule-based, similar to general sentiment analysis. The majority of research has focused on polarity ranking: Xia et al. provided a multi-step technique to user opinion classification.

Their proposed approach identifies the subject and the polarity being expressed towards that subject. An Fmeasure of about 0.67 was also reported. For the classification of sentiment in tweets, Sokolova et al. used a variety of classifiers, including Naive Bayes, decision trees, and support vector machines (SVMs). The texts were regarded as a jumble of words. Two clustering tasks were considered: three classes (positive, negative, and neutral) and two groups (positive, negative, and neutral) (positive, negative). An SVM classifier had the best Fmeasure of 0.69 .

The purpose of the work by Biyani et al. was to find the polarity of the expressed sentiments of patients in online health resources communities. In particular, they used the domain dependent and domain independent sentiments characteristics as the two complementary points of view of a message and used them for message classification in a semi-supervised setting using a co-training algorithm to complete a classification of user message sentiments in an online cancer support service community.

This effort was later improved with features that are derived from a dynamic sentiment vocabulary, while the earlier work employed a more general sentiment lexicon to abstract the patterns. Smith et al. studied an additional perspective of the sentiment in patient feedback, namely speech features like expressivity and persuasiveness. A classifier was tested on a selected corpus of patients' feedback from NHS Choices. The outcomes show that the Naïve Bayes multinomial classification with features based on frequency can obtain the best accuracy $(83.53 \%)$. In addition, the performance results demonstrated that a classifier model that was trained only on an expression corpus can be implemented directly on the persuasive corpus and achieve performance comparable to that of the corpus-based training with the same speech feature.

\section{MEDICAL SENTIMENT ANALYSIS: THE FUTURE}

In conclusion, the study of medical texts (medical social media texts, clinical accounts) with regards to the analysis of sentiment includes the possible need to meet the following research challenges:

- Modeling the implicit clinical context and identification of the implicit sentiment,

- Construction of a field-specific vocabulary of sentiment, - Context-specific determination of sentiment, and

- Modeling various aspects of the condition of the patient. Some other aspects are the identification of the view carrier and the incorporation of time.

An operation may start usually, but may become crucial. Because of this, the health feeling must also be regarded in time. Time is supplied by the document timestamp or sometimes in the documents themselves, or clinical data can be sorted into phases of treatment. As other research has already shown, negative statements are used very much in clinical narratives. In the sentiment analysis, it is very important to detect the use of negations because the polarity can be switched (e.g., in no complaint of pain when asked).

\section{CONCLUSIONS}

This article presents the various ways in which the subject of sentiment is used in the field of medicine and identifies areas for future study in the areas of medical sentiment analysis. While the existing work

analysis of sentiment from medicinal texts has focused on medical social media and biomedical literature, future work should also focus on the analysis of clinical documents. This analysis could be used for numerous purposes.

Uncertainty, attitudes, and implicit emotions might all be collected and used into therapeutic decision-making. The 
development of a domain-specific sentiment lexicon and techniques to judging sentiment based on the nature of the content could be the initial steps toward solutions for sentiment analysis.

\section{References}

1. Wiebe J, Bruce R. Probabilistic classifiers for tracking point of view. Progress in communication sciences 1995:125-142 [FREE Full text]

2. Hatzivassiloglou V, McKeown KR. Predicting the Semantic Orientation of Adjectives. In: Proceedings of the 35th Annual Meeting of the Association for Computational Linguistics and Eighth Conference of the European Chapter of the Association for Computational Linguistics. 1997 Presented at: ACL'98/EACL'98; July 7-12, 1997; Madrid, Spain p. $174-$

181 URL: https://www.aclweb.org/anthology/P971023/ [CrossRef]

3. Wiebe JM, Bruce RF, O'Hara TP. Development and Use of a Gold-standard Data Set for Subjectivity Classifications. In: Proceedings of the 37th annual meeting of the Association for Computational Linguistics on Computational Linguistics. 1999 Presented at: ACL'99; June 20-26, 1999; College Park, Maryland, USA p. 246253 URL: https://www.aclweb.org/anthology/P991032/ [CrossRef]

4. $\mathrm{Hu}$ M, Liu B. Mining Opinion Features in Customer Reviews. In: Proceedings of the 19th national conference on Artifical intelligence. 2004 Presented at: AAAI'04; July 25 - 29, 2004; San Jose, California, USA p. 755760 URL: https://dl.acm.org/citation.cfm?id=1597 269

5. $\mathrm{Hu}$ M, Liu B. Mining and Summarizing Customer Reviews. In: Proceedings of the tenth ACM SIGKDD international conference on Knowledge discovery and data mining. 2004 Presented at: KDD'04; August 22 - 25, 2004; Seattle, Washington, USA p. 168177 URL: https://dl.acm.org/citation.cfm?id=1014 073 [CrossRef]

6. Bollen J, Mao H, Zeng X. Twitter mood predicts the stock market. J Comput Sci 2011;2(1):1-8. [CrossRef]

7. Efron M. Cultural orientation: Classifying subjective documents by cociation analysis. In: Proceedings of the AAAI Fall Symposium on Style and Meaning in Language, Art, and Music. 2004 Presented at: AAAI'04; July 25-29, 2004; San Jose, California p. 41-48.

8. Ramteke J, Shah S, Godhia D, Shaikh A. Election Result Prediction Using Twitter Sentiment Analysis. In: Proceedings of the 2016 International Conference on Inventive Computation Technologies. 2016 Presented at: ICICT'16; August 26-27, 2016; Coimbatore, India p. 1-5. [CrossRef]

9. World Health Organisation. Geneva, Switzerland: World Health Organisation; 2006. Constitution of the
World

Health

Organisation URL: https://www.who.int/governanc e/eb/who constitution en.pdf [accessed 2019-1112]

10. Huber M, Knottnerus JA, Green L, van der Horst H, Jadad AR, Kromhout D, et al. How should we define health? Br Med J 2011 Jul 26;343:d4163. [CrossRef] [Medline]

11. Berg O. Health and quality of life. Acta Sociologica 1975;18(1):3-22. [CrossRef]

12. Afyouni S, Fetit AE, Arvanitis TN. \#DigitalHealth: exploring users' perspectives through social media analysis. Stud Health Technol Inform 2015;213:243246. [Medline]

13. Eysenbach G. Infodemiology and infoveillance: framework for an emerging set of public health informatics methods to analyze search, communication and publication behavior on the Internet. J Med Internet Res 2009 Mar 27;11(1):e11 [FREE Full text] [CrossRef] [Medline]

14. Eysenbach G. Infodemiology and infoveillance tracking online health information and cyberbehavior for public health. Am J Prev Med 2011 May;40(5 Suppl 2):S154-S158. [CrossRef] [Medline]

15. Rozenblum R, Bates D. Patient-centred healthcare, social media and the internet: the perfect storm? BMJ Qual Saf 2013 Feb 01;22(3):183-186. [CrossRef]

16. Ofcom. 2015. The communications market report URL: http://stakeholders.ofcom.org.uk/binar ies/research/cmr/cmr15/icmr15/icmr_2015.pdf [Web Cite Cache]

17. Lunden I. Techcrunch. 2013. Mobile twitterm+ (75\%) access from handheld devices monthly, $65 \%$ of ad sales come from mobile URL: http:/ /techcrunch.com/2013/10/03/mobile-twitter-161maccess-from-handheld-devices-each-month-65-ofad-revenues-coming-from-mobile/ [accessed 201604-13] [WebCite Cache]

18. Pang B, Lee L. Opinion mining and sentiment analysis. Foundations and trends in information retrieval 2008 Jul;2(1-2):1-35.

19. Liu B, Zhang L. A survey of opinion mining and sentiment analysis. Mining Text Data 2012:415-463. [CrossRef]

20. Nasukawa T. Sentiment analysis: capturing favorability using natural language processing. 2003 Jan 01 Presented at: Proceedings of the 2nd International Conference on Knowledge; October 2325, 2003; Sanibel Island, FL, USA. [CrossRef]

21. Chew C, Eysenbach G. Pandemics in the age of twitter: content analysis of Tweets during the 2009 H1N1 outbreak. PLoS One 2010 Nov 29;5(11):e14118 [FREE Full text] [CrossRef] [Medline]

22. Mohammad S. 9 - Sentiment analysis: detecting valence, emotions, and other affectual states from text. Emotion Measurement 2016:201-237. [CrossRef] 
23. $\mathrm{Hu} \mathrm{M}$, Liu B. Mining and summarizing customer reviews. In: Proceedings of the 10th ACM SIGKDD international conference on knowledge discovery and data mining, KDD '04. New York, NY, USA: ACM; 2004. p. 168-77

24. Mishne G. Experiments with mood classification in blog posts. In: 1st Workshop on stylistic analysis of text for information access. 2005.

25. Baldoni M, Baroglio C, Patti V, Rena P. From tags to emotions: ontology-driven sentiment analysis in the social semantic web. Intell Artif 2012;6(1):41-54

26. Pang B, Lee L, Vaithyanathan S. Thumbs up?: sentiment classification using machine learning techniques. In: Proceedings of the ACL-02 conference on empirical methods in natural language processing - Volume 10, EMNLP '02. Stroudsburg, PA, USA: Association for Computational Linguistics; 2002. p. 79-86.

27. Pang B, Lee L. A sentimental education: sentiment analysis using subjectivity summarization based on minimum cuts. In: Proceedings of the 42nd annual meeting on association for computational linguistics, ACL '04. Stroudsburg, PA, USA: Association for Computational Linguistics; 2004

28. Turney PD. Thumbs up or thumbs down?: Semantic orientation applied to unsupervised classification of reviews. In: Proceedings of the 40th annual meeting on association for computational linguistics, ACL '02. Stroudsburg, PA, USA: Association for Computational Linguistics; 2002. p. 417-24

29. Pang B, Lee L, Vaithyanathan S. Thumbs up?: sentiment classification using machine learning techniques. In: Proceedings of the ACL-02 conference on empirical methods in natural language processing - Volume 10, EMNLP '02. Stroudsburg, PA, USA: Association for Computational Linguistics; 2002. p. 79-86

30. Pontiki M, Galanis D, Pavlopoulos J, Papageorgiou $\mathrm{H}$, Androutsopoulos I, Manandhar S. Semeval-2014 task 4: aspect based sentiment analysis. In: Proceedings of the 8th international workshop on semantic evaluation (SemEval 2014). August 2014. p. $27-35$

31. Denecke K. Are sentiwordnet scores suited for multidomain sentiment classification? Proceedings of the fourth international conference on digital information management, 2009. ICDIM 2009. 2009. p. 1-6

32. Montejo-Ráez A, Martínez-Cámara E, MartínValdivia MT, López LAUrena. Random walk weighting over sentiwordnet for sentiment polarity detection on twitter. In: Proceedings of the 3rd workshop in computational approaches to subjectivity and sentiment analysis, WASSA '12.
Stroudsburg, PA, USA: Association for Computational Linguistics; 2012. p. 3-10.

33. Baccianella S, Esuli A, Sebastiani F. Sentiwordnet 3.0: an enhanced lexical resource for sentiment analysis and opinion mining. In: Calzolari N (Conference Chair), Choukri K, Maegaard B, Mariani J, Odijk J, Piperidis S, Rosner M, Tapias D, editors. Proceedings of the seventh international conference on language resources and evaluation (LREC'10). Valletta, Malta: European Language Resources Association (ELRA); 2010.

34. Balahur A, Steinberger R, Kabadjov MA, Zavarella V, Van der Goot E, Halkia M, et al. Sentiment analysis in the news. Comput Res Repos (CoRR) 2013, abs/1309.6202

35. Wilson T, Wiebe J, Hoffmann P. Recognizing contextual polarity in phraselevel sentiment analysis. In: Proceedings of the conference on human language technology and empirical methods in natural language processing, HLT '05. Stroudsburg, PA, USA: Association for Computational Linguistics; 2005. p. 347-54

36. Mohammad SM, Turney PD. Emotions evoked by common words and phrases: Using mechanical turk to create an emotion lexicon. In: Proceedings of the NAACL HLT 2010 workshop on computational approaches to analysis and generation of emotion in text, CAAGET '10. Stroudsburg, PA, USA: Association for Computational Linguistics; 2010. p. 26-34.

37. Deng L, Choi Y, Wiebe J. Benefactive/malefactive event and writer attitude annotation. In: Proceedings of the 51st annual meeting of the association for computational linguistics (Volume 2: Short Papers). Sofia, Bulgaria: Association for Computational Linguistics; August 2013. p. 120-5

38. Goeuriot L, Na J-C, Min Kyaing WY, Khoo C, Chang Y-K, Theng Y-L, et al. Sentiment lexicons for health-related opinion mining. In: Proceedings of the 2Nd ACM SIGHIT international health informatics symposium, IHI '12. New York, NY, USA: ACM; 2012. p. 219-26.

39. Ohana B, Tierney B, Delany S. Domain independent sentiment classification with many lexicons. In: 2011 IEEE workshops of international conference on advanced information networking and applications (WAINA). March 2011. p. 632-7

40. A. Mecke, I. Lee, J.R. Baker jr., M.M. Banaszak Holl, B.G. Orr, Eur. Phys. J. E 14, 7 (2004)

41. M. Ben Rabha, M.F. Boujmil, M. Saadoun, B. Bessaïs, Eur. Phys. J. Appl. Phys. (to be published)

42. L. T. De Luca, Propulsion physics (EDP Sciences, Les Ulis, 2009)

43. F. De Lillo, F. Cecconi, G. Lacorata, A. Vulpiani, EPL, 84 (2008)

44. G. Plancque, D. You, E. Blanchard, V. Mertens, C. Lamouroux, Role of chemistry in the phenomena 
occurring in nuclear power plants circuits, in

Proceedings of the International Congress on

Advances in Nuclear power Plants, ICAPP, 2-5

May 2011, Nice, France (2011)

45. K.Denecke, Y.Deng, Sentiment analysis in medical settings: New opportunities and challenges, Artificial Intelligence in Medicine, march 2015. 